\title{
Single chain elasticity and thermoelasticity of polyethylene
}

\author{
John T. Titantah ${ }^{1,2 *}$, Carlo Pierleoni ${ }^{1}$ and Jean-Paul Ryckaert ${ }^{2}$ \\ ${ }^{1}$ INFM and Dipartimento di Fisica, Università degli studi, \\ Via Vetoio, I-67100 L’Aquila, Italy \\ ${ }^{2}$ Unité de Physique des Polymères, CP 223, Université Libre de Bruxelles, \\ Bd du Triomphe, B-1050 Brussels, Belgium
}

November 3, 2018

\begin{abstract}
Single-chain elasticity of polyethylene at $\theta$ point up to $90 \%$ of stretching with respect to its contour length is computed by Monte-Carlo simulation of an atomistic model in continuous space. The elasticity law together with the free-energy and the internal energy variations with stretching are found to be very well represented by the wormlike chain model up to $65 \%$ of the chain elongation, provided the persistence length is treated as a temperature dependent parameter. Beyond this value of elongation simple ideal chain models are not able to describe the Monte Carlo data in a thermodynamic consistent way. This study reinforces

\footnotetext{
${ }^{*}$ Present address: Theoretical Study of Matter, Department of Physics, University of Antwerpen, Groe-
} nenborgerlaan 171, 2020 Antewerpen (Belgium)
\end{abstract}


the use of the wormlike chain model to interpret experimental data on the elasticity of synthetic polymers in the finite extensibility regime, provided the chain is not yet in its fully stretched regime. Specific solvent effects on the elasticity law and the partition between energetic and entropic contributions to single chain elasticity are investigated. 


\section{Introduction}

When a single linear chain of $\mathrm{N}$ segments is stretched at both ends by equal and opposite forces $\pm \underline{f}$, the average end-to-end vector $\langle\underline{R}>$ lies in the direction of the force and its magnitude $R$ is related to $f$ by an elasticity law or equation of state (EOS) that is usually casted in the dimensionless form

$$
\frac{f \ell}{k_{B} T}=w(R, N, T)
$$

where $k_{B}$ is the Boltzmann constant, $\mathrm{T}$ the absolute temperature, $\ell$ a characteristic length of the unstressed chain and $w$ a dimensionless function.

At very low force intensities the equation of state follows the simple linear Hooke's law |11

$$
\frac{f R_{0}}{k_{B} T}=3 \frac{R}{R_{0}}
$$

where $R_{0}$ is the end-to-end vector (in the mean square sense) of the polymer in its unstressed state. For somehow larger forces $\left(f R_{0} / k_{B} T \geq 2\right)$ the so called "Pincus" regime is entered[3, 2]

$$
\frac{f R_{0}}{k_{B} T} \sim\left(\frac{R}{R_{0}}\right)^{\gamma}
$$

where $\gamma=1,3 / 2$ for $\theta$ and good solvent conditions respectively [1]. Note that for $\theta$ chains the two regimes merge in a single one. The Pincus regime is abandoned in favor of the so called "Finite Extensibility" regime (FE) when the applied force is further increased beyond $f b_{k} / k_{B} T=1$ where $b_{k}$, the Kuhn segment of the polymer, is a measure of the local rigidity of the chain. In this FE regime, the EOS depends on the microscopic details of the specific chain being stretched and, for long enough chains, the EOS can be generally written

$$
\frac{f b_{k}}{k_{B} T}=w(X, T)
$$

where $X=R / L_{c}$ and $L_{c}$ is the chain contour length.

Elasticity laws of real chains in this regime are usually discussed in terms of simple ideal

models like the Freely Jointed chain (FJC), the Wormlike chain (WLC) and some ad hoc 
extensions (elastic FJC and elastic WLC) [4, 6, 6, 7, 8]. The great success of the WLC in describing the elasticity of double-stranded DNA and of other biopolymers [6] suggests the existence of some form of universality in the FE regime also. In general the ideal chain models predict an EOS of the form (四) with a temperature independent right hand side $w=w(X)$

$$
\frac{f b_{k}}{k_{B} T}=w(X)
$$

In eq. (5), $b_{k}$ is usually treated as a constant, the force being thus proportional to the temperature at any fixed relative extension of the chain, in good agreement with the well known thermo-elasticity properties of rubbers. However, the variation with temperature of the size of the polymer coil in a melt [9, 10] implies a temperature dependence of $b_{k}=$ $R_{0}^{2} / L_{c}$, meaning that the local rigidity of the chain is affected by the temperature. In the context of single chain elasticity, the ideal chain models EOS eq. (国) are used to interpret the experimental data on the $X$ dependence of the force. The Kuhn segment $b_{k}$ and the contour length $L_{c}$ are considered as fitting parameters in the analysis, but the temperature dependence of $b_{k}$ cannot be inferred since experiments are usually performed isothermally at a unique temperature [5, 11, 12, 13]. When attention is payed to energetic aspects of the stretching, the temperature dependence of $b_{k}$ becomes a sensible point. If the chain EOS follows eq. (5) with $b_{k}$ constant, thermoelasticity predicts that the internal energy of the chain does not change with stretching and equivalently, that the internal force has a pure entropic character $\left(f=f_{S}\right)$. The same EOS eq. (5) with a temperature dependent $b_{k}$ predicts that the internal energy change due to isothermal stretching is proportional to the corresponding free energy change over the whole range of stretching and the ratio $f_{S} / f(\neq 1)$ is independent of stretching. Therefore, ideal models with temperature dependent Kuhn segment have the whole thermodynamic complexity, and are very promising candidates to interpret real chain behaviors.

The temperature dependence of the Kuhn segment has been much investigated experi- 
mentally for a large class of synthetic polymers [9] and more particularly for polyethylene (PE) (see ref [10] and references therein). It has been obtained from the temperature dependence of the radius of gyration of the chain in melts, from intrinsic viscosity measurements of the polymer in various $\theta$ point solvents or from thermoelasticity measurements on polymer networks. For PE, the chain dimension in the $\theta$ state is found to increase with decreasing temperature. On the basis of Rotational Isomeric State (RIS) model calculations, this trend has been related to the increase of trans population (respect to gauche) of the dihedral angles of the chain backbone as temperature drops [14 which leads to a local straightening of the chain reflected by an increase of the length of the Kuhn segment.

On this basis it is tempting to test more deeply the validity of ideal models to represent real chain thermo-elasticity by measuring single chain elasticity law for synthetic polymers. Experimentally, the interpretation of such elasticity data is made difficult by the limited signal to noise ratio and the limited range of forces accessible which hamper an accurate data fitting [11, 8, 7].

A possible alternative route to investigate single chain elasticity is provided by numerical simulation of theoretical models, either coarse grained or atomistic [15, 16, 17, 18, 19, 20, 21]. In the present work we use the Monte-Carlo (MC) simulation approach on a well defined polymer atomistic model to establish with accuracy the chain elasticity law over a wide range of extension $\left(0<R / L_{c}<0.9\right)$ including the finite extensibility regime and the corresponding change in the chain internal energy with stretching. Our aim is twofold: i) to assess the validity of an EOS of the type of eq. (5) definitely simpler than the general form of eq. (田); ii) to test the applicability of simple ideal models to represent $\mathrm{MC}$ data. To follow this program, we consider the synthetic polymer with the simplest microscopic structure, namely $\mathrm{PE}$ and we adopt an atomistic model in continuous space. To access both weak and high stretching regimes with good statistical accuracy, we exploit the configurational bias MC methodology in combination with biased fixed- $f$ ensemble sampling techniques. 
The thermoelasticity of PE has been the subject of a previous Monte Carlo study for a lattice model [15] but that work is focused on the study of the solvent effect more than on the EOS and the test of ideal chain models.

The paper is organized as follows: in the next section, we review the theoretical framework of single chain thermo-elasticity and its formal statistical mechanics basis. With the aim of performing single chain simulations, we review the theoretical status of a single chain models meant to be representative of a chain embedded in a solvent (or in a bath of similar

chains) for which intramolecular potentials have a free energy character. In section III, we give for completeness the adopted PE model, namely the united-atom model devised originally by A. Sariban et al. [22, 23]. We give in section IV some details about the PE simulations performed within the fixed force ensemble, the results of which can be used to get also averages in the fixed end-to-end vector ensemble. In section $\mathrm{V}$, we analyze the results for $\mathrm{PE}$ chains, most of them being relative to $\theta$ point (melt state) at $400 \mathrm{~K}$ while a few data are presented for PE in good solvent. We discuss the free energy and internal energy evolutions with stretching in the FE regime, looking at the partition between the various energy contributions. We analyze the adequacy of the FJC and WLC models to represent the thermo-elastic behavior of PE. Section VI is concerned with some results and a short discussion on the effects of excluded volume on the elasticity law of PE. Section VII gathers our conclusions.

\section{Theoretical framework}

\subsection{Thermodynamics}

The central quantity in single chain thermo-elasticity is the chain Helmholtz free energy $A(R, T)$ the total differential being

$$
d A=-S d T+\underline{f} \cdot d \underline{R} .
$$


The corresponding single chain internal energy is then given by

$$
E(\underline{R}, T)=\left(\frac{\partial \beta A(\underline{R}, T)}{\partial \beta}\right)_{\underline{R}}
$$

where $\beta=1 / k_{B} T$. The internal force existing within a stretched chain with end-to-end vector $\underline{R}$ can naturally be split into an entropic $\left(\underline{f}_{S}\right)$ and an energetic part $\left(\underline{f}_{E}\right)$ according to

$$
\underline{f}(\underline{R}, T)=\left(\frac{\partial A(\underline{R}, T)}{\partial \underline{R}}\right)_{T}=\underline{f}_{E}+\underline{f}_{S}
$$

where

$$
\underline{f}_{E}=\left(\frac{\partial E(\underline{R}, T)}{\partial \underline{R}}\right)_{T}=-T^{2}\left(\frac{\partial[\underline{f}(\underline{R}, T) / T]}{\partial T}\right)_{\underline{R}}
$$

and

$$
\underline{f}_{S}=-T\left(\frac{\partial S(\underline{R}, T)}{\partial \underline{R}}\right)_{T}=T\left(\frac{\partial \underline{f}(\underline{R}, T)}{\partial T}\right)_{\underline{R}}
$$

\subsection{Statistical Mechanics}

The system under study is a single homo-polymer chain, with degree of polymerization $N$, which is surrounded by a bath of similar (melt case) or dissimilar (solvent) molecules (note that in the following $N$ will always represent the number of skeletal bonds in the linear chain). To simplify notations, the $N$ dependence of single chain quantities will not be made explicit in the following formal developments.

The link between the Helmholtz free energy $A(\underline{R}, T)$ and statistical mechanics is

$$
A(\underline{R}, T)=-k_{B} T \log Z_{R}(\underline{R}, T)
$$

where the partition function $Z_{R}(\underline{R}, T)$, appropriate to a fixed- $R$ ensemble (hence the $R$ index to emphasize the nature of constraint), is expressed as

$$
Z_{R}(\underline{R}, T)=\int d \underline{\Gamma}_{1} \delta\left(\underline{r}_{N}-\underline{r}_{0}-\underline{R}\right) \exp \left(-\beta \tilde{U}\left(\underline{\Gamma}_{1} ; T, \rho\right)\right)
$$

where $\underline{\Gamma}_{1}$ is the set of cartesian coordinates $\left(\underline{r}_{i}\right)_{i=0, N}$ of the $N+1$ point particles of the chain. In this equation, $\tilde{U}\left(\underline{\Gamma}_{1}\right)$ is the effective potential (or potential of mean force) characterizing 
the chain embedded in a bath consisting of the other chains of the melt or the solvent in an infinite dilution situation. The bath is characterized by a temperature $T$ and melt or solvent density $\rho$ and therefore the effective potential $\tilde{U}\left(\underline{\Gamma}_{1}\right)$ originates from an integration over the (infinitely large number of) bath degrees of freedom $\underline{\Gamma}_{2}$, namely

$$
\exp \left(-\beta \tilde{U}\left(\underline{\Gamma}_{1} ; T, \rho\right)\right) \equiv \int d \underline{\Gamma}_{2} \exp \left(-\beta U\left(\underline{\Gamma}_{1}, \underline{\Gamma}_{2}\right)\right)
$$

where $U\left(\underline{\Gamma}_{1}, \underline{\Gamma}_{2}\right)$ is the bare total potential of the chain + bath system.

We further note the well known equivalence

$$
Z_{R}(\underline{R}, T)=Z(T) W^{0}(R, T)
$$

where $Z(T)=\int d \underline{R} Z_{R}(\underline{R}, T)$ is the partition function of the free chain in the bath at temperature $\mathrm{T}$ and $W^{0}(\underline{R}, T) d \underline{R}$ is the probability to find the end-to-end vector of that free chain in the range between $\underline{R}$ and $\underline{R}+d \underline{R}$.

Any single chain property $O(\underline{R}, T)$ (such as the tensile internal force or any structural quantity like a bond-alignment factor) is an average computed over the subset of configurations of the free chain which satisfy a fixed- $R$ value. More explicitly, the average of the microscopic quantity $\hat{O}$, denoted as $\langle\hat{O}\rangle_{R}$, reads

$$
O(\underline{R}, T) \equiv<\hat{O}>_{R}=Z_{R}(\underline{R}, T)^{-1} \int d \underline{\Gamma}_{1} \delta\left(\underline{r}_{N}-\underline{r}_{0}-\underline{R}\right) \hat{O}\left(\underline{\Gamma}_{1}\right) \exp \left(-\beta \tilde{U}\left(\underline{\Gamma}_{1} ; T, \rho_{s}\right)\right)
$$

The corresponding average $O(T)$ for the free chain is related to the fixed- $R$ averages through the relationship

$$
O(T)=\int d \underline{R} W^{0}(R, T) O(R, T)
$$

We emphasize here the special case of the internal energy $E(R, T)$ which can be expressed as an average quantity of the type of eq.(15). Indeed, due to the free energy nature (temperature dependency) of the potential, one gets by combining basic eqs. (17),(11),(12) and (13) the expression

$$
E(\underline{R}, T)=<\frac{\partial(\beta \tilde{U})}{\partial \beta}>_{R}
$$




\section{Model of polyethylene chain}

Our purpose is to extract the elasticity law of a PE model which is sufficiently realistic to be pertinent to that specific polymer but which avoids explicit consideration of the bath degrees of freedom. This is done by modeling the system of interest at the level of the $\tilde{U}\left(\underline{\Gamma}_{1}\right)$ function for which we adopt the Sariban model [22, 23]. In this model based on methylene "united" atoms, the carbon-carbon bonds and skeletal bending angles are both rigid with bond lengths set to $d_{c c}=1.54$ angstrom and bending angle set to $\gamma=109.7$ degrees. These geometrical data are required to evaluate the contour length $L_{c}$ of the PE chain with $N$ C-C bonds according to

$$
L_{c}=N d_{c c} \sin (\gamma / 2)
$$

The intra-molecular potential, written for a chain with $\mathrm{N}+1$ methylene groups, is split into a sum of local contributions and non local interactions,

$$
\tilde{U}\left(\underline{\Gamma}_{1}\right)=U^{l o c}+U^{\text {nonloc }}
$$

where

$$
U^{l o c}=\sum_{i=2}^{N-1} V^{t o r}\left(\phi_{i}\right)+\sum_{i=0}^{N-4} v_{L J}\left(\left|\underline{r}_{i+4}-\underline{r}_{i}\right|\right)
$$

contains single bond torsion contributions $V^{\text {tor }}$ of the "n-butane" type taken from the "CHARMM" force field [24, 25] and 1-5 interactions coping with the pentane effect [9]. The non-local potential part is built as a sum of effective pairwise interactions of the LennardJones type between united atoms at least fifth neighbor along the polymer contour,

$$
U^{\text {nonloc }}=\sum_{i=0}^{N-5} \sum_{j=i+5}^{N} 4 \epsilon^{\star}\left[\left(\frac{\sigma^{\star}}{r_{i j}}\right)^{12}-\lambda\left(\frac{\sigma^{\star}}{r_{i j}}\right)^{6}\right] .
$$

The attractive part of the pair interactions is modulated by a factor $\lambda$ allowing the solvent quality to be varied by adjusting the relative weight of repulsive and attractive interactions. Lennard-Jones parameters for the present PE model are $\epsilon^{\star}=0.4301 \mathrm{KJ} / \mathrm{mol}$ and $\sigma^{\star}=3.74 \AA$. 
For a given temperature T, our PE chain will be at $\theta$ point for a particular value $\lambda=\lambda^{\star}(T)$ defined as the $\lambda$ value which guarantees that

$$
\lim _{N \rightarrow \infty}\left|\left\langle\left(\underline{r}_{N}-\underline{r}_{0}\right)^{2}\right\rangle\right|_{\lambda=\lambda^{\star}} \equiv \lim _{N \rightarrow \infty}\left|\left\langle R_{0}^{2}\right\rangle\right|_{\lambda_{=\lambda^{\star}} \sim N}
$$

leading to the Flory characteristic ratio $C_{\infty}$ defined by

$$
\lim _{N \rightarrow \infty} \frac{1}{\left(N \ell_{c c}^{2}\right)}\left|\left\langle R_{0}^{2}\right\rangle\right|_{\lambda=\lambda^{\star}} \equiv C_{\infty}(T)
$$

At $\mathrm{T}=400 \mathrm{~K}$, it was found that $\lambda^{\star}=0.505 \pm 0.005$ and $C_{\infty}=7.9 \pm 0.2$ [25]. This value of $\lambda^{\star}$ differs slightly from the value $\lambda^{\star}=0.53$ quoted in reference [23] which was obtained at the same temperature with much shorter chains and a slightly different n-butane torsional form. In the same work [23], the temperature dependence of $\lambda^{\star}$ for the PE model at $\theta$ point was estimated to be

$$
\frac{\lambda^{\star}(T)}{T}=\frac{0.2}{T}+0.0008 K^{-1}
$$

Performing an equilibrium sampling of the PE chain with the potential $\tilde{U}\left(\underline{\Gamma}_{1}\right)$ yields an energy at fixed $\underline{R}$ given by eq.(17) where

$$
\frac{\partial \beta \tilde{U}}{\partial \beta}=V^{l o c}+\sum_{i=0}^{N-5} \sum_{j=i+5}^{N} 4 \epsilon^{\star}\left[\left(\frac{\sigma^{\star}}{r_{i j}}\right)^{12}-\frac{\partial \beta \lambda^{\star}}{\partial \beta}\left(\frac{\sigma^{\star}}{r_{i j}}\right)^{6}\right]
$$

in which, according to eq. 24), $\partial\left(\beta \lambda^{\star}\right) / \partial \beta=0.2$.

The internal energy of the PE chain can be rewritten as a sum of potential contributions,

$$
E(R, T)=E^{l o c}(R, T)+E^{r e p}(R, T)+\frac{\partial \beta \lambda^{\star}}{\partial \beta} E^{a t t}(R, T)
$$

where the three terms are the local potential contribution, due to torsion plus the 1-5 interactions, the repulsive and the attractive parts of non local contributions respectively. They are ensemble averages of the corresponding terms in eq.(25). 


\section{Computational methodology}

Single chain Monte-Carlo simulations of PE have been performed by the configurational bias Monte-Carlo (CBMC) method [26], in combination with reptation moves of a few methylene units. Such a numerical procedure is relatively simple as long as the chain is free of any constraint on the end to end vector. We explain below how the method works for a free chain and how it can be adapted for sampling a fixed- $f$ chain ensemble, i.e. when a pair of equal and opposite stretching forces are applied at both chain ends. Using end to end distance histogram analysis, these fixed stress ensemble samplings are shown to yield the fixed- $R$ ensemble data which are more appropriate to discuss the thermo-elastic properties of chains.

\subsection{Sampling in the fixed-f single chain ensemble}

For free chains (not subjected to external forces), a sequence of $k$ bonds is grown at one end of the chain (the particular end being chosen at random) while a sequence of $k$ bonds is suppressed at the other end. Individual dihedral angles (the only relevant variables) associated with the growth process are generated according to a distribution $\propto \exp \left(-\beta V_{\text {tor }}\right)$, the rest of the interactions influencing the acceptance rate through the Rosenbluth weight [26].

When simulating chains with an external stretching force along the $\mathrm{z}$ axis $(0,0, \pm f)$, the associated external potential energy can be taken into account in the dihedral angle generation by sampling a distribution $\propto \exp \left(-\beta\left(V_{t o r}-z f\right)\right)$ where $\mathrm{z}$ is the projection of the new bond in the direction of the external force. Alternatively this external energy contribution can be taken into account in the Rosenbluth weight. We found that the latter method works well as long as the force is not too strong $\left(f<k_{B} T / b_{k}\right)$ but that it is advantageous to switch to the former method when the force becomes higher.

For a $\mathrm{PE}$ chain of $\mathrm{N}$ bonds sampled by $\mathrm{MC}$ with acceptance probability $P_{\text {acc }}$ for the 
individual CBMC/reptation moves of $k$ bonds, the generation of a new independent configuration typically requires $\left[(N / 2 k)^{2} P_{a c c}^{-1}\right] \mathrm{MC}$ attempted steps [17. We did experiments for $\mathrm{T}=400 \mathrm{~K}$ using $\lambda=\lambda^{\star}(400)=0.505$ for $\mathrm{N}=256$ and 512 which model $\theta$ point conditions [25]. A few runs in good solvent at the same temperature were performed with $\lambda=0.01$ for $\mathrm{N}=256$. All results have been produced with $k=8$ methylene units for the CBMC/reptation moves. A minimum of about 20.000 independent configurations have been generated in each experiment. Such a large sampling was motivated by the need of a high statistical accuracy in the histogram analysis to be described below.

\subsection{Fixed-R ensemble results}

Fixed- $R$ ensemble results can easily be obtained from a fixed- $f$ ensemble sampling using histograms based on the instantaneous $R$ value of the sampled stressed chain.

Let us first remind that the fixed- $f$ fixed- $T$ ensemble is characterized by a partition function $Z_{f}$ defined as

$$
Z_{f}(f, T)=\int d \mathbf{r}^{N} \exp \left[-\beta\left(U\left(\mathbf{r}^{N}\right)-\left(\mathbf{r}_{N}-\mathbf{r}_{0}\right) \cdot \mathbf{f}\right)\right]
$$

with the property that $Z_{f}(0, T)$ is equivalent to the partition function of a free chain $Z(T)$ already discussed in eq. (14). Let $\langle\hat{O}\rangle_{f}$ denote the average of an arbitrary microscopic quantity $\hat{O}$ in that ensemble.

Let us now introduce the probability $W^{f}(\underline{R}, T) d \underline{R}$ to find the end-to-end vector of the chain in the range $(\underline{R}, \underline{R}+d \underline{R})$ in the stressed chain ensemble defined by eq.(27). We have

$$
W^{f}(\mathbf{R}, N)=\frac{\int d \mathbf{r}^{N} \delta\left(\mathbf{r}_{N}-\mathbf{r}_{0}-\mathbf{R}\right) \exp \left[-\beta\left(U\left(\mathbf{r}^{N}\right)-\left(\mathbf{r}_{N}-\mathbf{r}_{0}\right) \cdot \mathbf{f}\right)\right]}{Z_{f}}
$$

The distribution of the end-to-end vector in the absence of force, i.e. $W^{0}(\mathbf{R}, N)$ introduced in eq. (14), is related to $W^{f}(\mathbf{R}, N)$ by

$$
W^{0}(\mathbf{R}, N)=\frac{Z_{f}}{Z} \exp (-\beta \mathbf{R} \cdot \mathbf{f}) W^{f}(\mathbf{R}, N)
$$


where the r.h.s. is globally $f$ independent and isotropic in $\mathbf{R}$ while $W^{f}(\mathbf{R}, N)$ depends on $f$ and has a cylindrical symmetry along the force direction. By superposing the distributions $W^{0}$ (known up to some multiplicative constant using eq. (29)) obtained from the histograms

of $W^{f}(\mathbf{R}, N)$ established for a set of simulations at several force intensities, the $W^{0}(R, T)$ profile can be obtained over a large range of $R$ values with relatively modest computational efforts.

In order to estimate $O(\underline{R}, T)$ as defined in eq. (15) from the sampling of the stressed chain ensemble, one exploits the relationship

$$
<\hat{O} \delta\left(\mathbf{r}_{N}-\mathbf{r}_{0}-\mathbf{R}\right)>_{f}=W^{f}(R, T)<\hat{O}>_{R}
$$

This expression means that $O(R, T) \equiv<\hat{O}>_{R}$ can be estimated within the fixed- $f$ ensemble sampling by a simple arithmetic average of the individual $\hat{O}$ values relative to all configurations with an end-to-end vector lying in the relevant box in the $R$ histogram.

In table 1, we list the characteristics of the different fixed- $f$ experiments which are exploited in this paper. In the last column, for each force value and for the indicated sampling quality, we mention the range of distances which has been exploited to reconstruct the unperturbed chain $W^{0}(R, T)$ profile according to eq.(29).

\section{Results}

\subsection{Elastic properties of the realistic PE model}

Figure 1 shows the force extension law for a $\theta$ chain of $\mathrm{PE}$ at $400 \mathrm{~K}$ obtained via fixed- $f$ ensemble calculations. The data used are, after transformation to reduced units, the points $\left(<\mathbf{R}>_{f}, f\right)$ obtained from individual simulation runs conducted for a specific combination of $f$ and $N$ values (see table (1). To emphasize the universal character ( $\mathrm{N}$ independent) of the law, we display the data corresponding to two chain lengths $\mathrm{N}=256$ and 512 in 
reduced variables, namely we plot the reduced force $f b_{k} / k_{B} T$ using the equilibrium estimate of $b_{k}=14.9$ angstrom 25 versus the reduced elongation $X=R / L_{c}$ with $L_{c}$ evaluated according to eq.(18). The force extension law in the fixed- $R$ ensemble (not shown) was also estimated using eq.(8) performing the derivative of a best fit function joining the free energy data of $\ln (\beta A(R, T))$ (to be discussed later) and the elasticity curve was found to be in close agreement with the fixed-f ensemble one, except in the $R<R_{0}$ region [27, 18] where the two ensembles strongly differ. (Note that the size of this $R / L_{c}$ pathological region decreases as $N^{-1 / 2}$ with chain length).

The data obtained for $N=256$ and $N=512$ yield a unique elasticity curve $w(X)$ which will be treated in the following as a 'quasi experimental' curve corresponding to the adopted 'realistic' $\theta$ chain $\mathrm{PE}$ model. We observe a linear regime up to $\approx 35 \%$ stretching which is followed by a rapid increase of the force due to chain FE. The discussion on the validity of simple chain elasticity models, like those relative to FJC and WLC models, to reproduce the MC data is postponed to a later section.

We first analyze how the internal energy of the chain and its various contributions evolve under stretching. According to eq.(26), the attractive part of the non local interactions must be weighted with the factor $\partial\left(\beta \lambda^{\star}\right) / \partial \beta$ which is quoted to be 0.2 by Sariban et al. 23] but that we took equal to 0.215 after the analysis detailed in section 6. To stress the universality of the curves, we reduce all energetic contributions by a factor $k_{B} T$ for dimensional reasons and by a factor $L_{c} / b_{k}$ to work with intensive quantities.

In figure 2 , we plot the deviations of various energetic quantities from their unperturbed value $\left(R=R_{0}\right)$ in the same thermodynamic conditions versus $X=R / L_{c}$. Data are shown for two chain lengths $N=256$ and $N=512$. Throughout this paper, the change of a quantity $Y$ with stretching will be denoted as $\Delta Y(R)$ and defined as $\Delta Y(R) \equiv Y(R)-Y\left(R_{0}\right)$.

For $\mathrm{N}=512$, the various contributions to the internal energy are shown on the same figure and we note that those arising from the single bond rotational energy largely dominate all 
other terms. The local $1-5$ interactions appear to have a marginal influence and, more interestingly, the non-local interactions contribute in a negligible way, in agreement with the $\theta$ point conditions imposed by the choice $\lambda=\lambda^{\star}$ in our simulations. We come back on these solvent quality aspects in section 6 where we show that good solvent conditions $\left(\lambda<<\lambda^{\star}\right)$ yield instead, as expected, a $R$-dependent additional contribution to the internal energy $E(R, T)$ of the chain.

\subsection{Rationalization of PE thermo-elasticity data}

The elasticity law shown in fig.1 for our realistic model of $\theta$ chains of PE at $400 \mathrm{~K}$ is a universal curve we will denote as $w(X, T)$. In the same figure, we compare these data with the functional forms relative to the FJC and of the WLC models which we denote respectively as $w_{F J C}(X)$ and $w_{W L C}(X) \cdot w_{F J C}(X)$ is simply the inverse Langevin function while the WLC curve $w_{W L C}(X)$ and its integral $W_{W L C}(X)$, which are required for the free energy calculation, were evaluated by the numerical procedure, accurate to $1.5 \%$, which is reported in eqs.(13)-(14) of ref.[6]. We want to emphasize that the curves in figure 11 are obtained using the values of $L_{c}$ and $b_{K}\left(\right.$ or $\left.\ell_{p}\right)$ obtained in the $\mathrm{MC}$ model at equilibrium and are not fitted to MC data. We note that the function $w(X, T)$ based on MC data and both simple models curves are all starting as $\approx 3 X$ at small $X$ and diverge (in different ways) as $X$ gets close to 1 .

The origin of elasticity in FJC and WLC models, and by extension to arbitrary models satisfying eq.(5), is often considered as purely entropic, a character which is commonly interpreted as the result of the reduction of the number of conformations as the chain extends. This is coherent with the functional form of the eq. (5) as long as the Kuhn segment $b_{k}$ (or equivalently the persistence length $\ell_{p}$ ) is constant, i.e. independent of temperature and external force. Eq.(5) then expresses that $f$ is proportional to $T$ at fixed relative extension,

which directly implies that the force is purely entropic (see eq.(9)). The absence of any 
energetic contribution to the force is thermodynamically equivalent to the independence of the internal energy respect to chain stretching, as indicated by eq.(9). When analyzing together fig. 2 and fig. 1, it appears that the variation of internal energy with stretching for PE cannot be avoided as soon as the finite extensibility regime is entered.

In the following, we show that PE elasticity can be modeled over a wide range of stretching including a large part of the finite extensibility regime on the basis of eq. (5) where $b_{k}$ is treated as a temperature dependent quantity. Such a temperature dependence of the persistence length (or Kuhn segment) has been invoked earlier in the case of the WLC model for DNA when it models a uniform elastic rod characterized by two parameters, namely the contour length $L_{c}$ and the elastic bending modulus $\kappa^{\prime}$ [28]. In this model, the persistence length, given by $\ell_{p}=\kappa^{\prime} / k_{B} T$, decreases with increasing temperature as a result of increasing Brownian undulations of the flexible rod. Here, for synthetic polymers, the temperature dependence of $b_{k}$ or $l_{p}$ must be reflected on the unperturbed size of the free polymer $R_{0}$ at $\theta$ point, given the relationships $R_{0}^{2}=L_{c} b_{k}=2 L_{c} \ell_{p}$.

The form of eq. (5) with a temperature dependent persistence length has some general implications on the elasticity law. If we denote by $W(X)$ the integral between 0 and $X$ of $w(X)$, we easily get the free energy and internal energy deviations from the unstressed state

$$
\begin{aligned}
\Delta A(R) & =\frac{L_{c} k_{B} T}{\ell_{p}} W\left(R / L_{c}\right) \\
\Delta E(R) & =\frac{L_{c} k_{B} T}{\ell_{p}} \frac{d \ln \left(\ell_{p}\right)}{d \ln (T)} W\left(R / L_{c}\right)=\frac{d \ln \left(\ell_{p}\right)}{d \ln (T)} \Delta A(R)
\end{aligned}
$$

Eq. (32) indicate that $\Delta E(R)$ and $\Delta A(R)$ are proportional. Using eqs. (8) and (9), the proportionality factor satisfies

$$
\frac{d \ln \left(\ell_{p}\right)}{d \ln (T)}=\frac{\Delta E(R)}{\Delta A(R)}=\frac{f_{E}}{f}
$$

which implies that the enthalpic/entropic partition of the total force is independent of stretching. 
In figure 3, eq.(32) is tested for two chain lengths $(N=256$ and $N=512)$. We indeed find a proportionality between the free energy change and the corresponding internal energy change over a stretching range going up to about 65\%, the best value for the proportionality factor being $d \ln \left(\ell_{p}\right) / d \ln (T)=-0.42$. Experimental values of $d \ln \left(R_{0}^{2}\right) / d T$ for $\mathrm{PE}$ in different $\theta$ solvents in the temperature range $(373 \mathrm{~K}-463 \mathrm{~K})$ are negative with modulus in the range $(1.0 \div 1.2) 10^{-3} K^{-1}[9]$. At $400 K$, this correspond to values of $d \ln \left(R_{0}^{2}\right) / d \ln (T)=$ $d \ln \left(\ell_{p}\right) / d \ln (T)$ in the range $-0.48 \div-0.40$ in agreement with our result. Previous calculations for PE at equilibrium performed at various temperatures on a very similar model lead to $d \ln \left(R_{0}^{2}\right) / d \ln (T)=-0.5 \pm 0.123$ in agreement with our finding based on stretching experiments.

The results shown in figure 3 lead to several important conclusions. The elasticity law of the PE model at $\theta$ state is well represented by a law of the type as in eq. (5), with a temperature independent r.h.s., up to a relative extension of $65 \%$. The variation of the internal energy in this range of elongations is provided by eq. (32). This elasticity law implies that, in the range of elongations below $65 \%$ which includes a large fraction of the finite extensibility regime, one has indeed constant ratios $f_{E} / f=-0.42$ and $f_{S} / f=1.42$. Beyond this regime of extension, the more general form of the elasticity law given in eq. (4) with a temperature dependent Kuhn segment, must be considered. Note that simple ideal model like FJC and WLC, even with temperature dependent Kuhn segment or persistence length, cannot provide elasticity laws of this kind.

The above picture on the thermo-elasticity of simple chain models such as PE reveals that, as the temperature decreases, the average size of the chain increases as a result of the relative increase of the population of trans conformers. In fig. 4 , we show the variation with stretching of the gauche population (expressed in percent) $\Delta n_{g}(R)=n_{g}(R)-n_{g}\left(R_{0}\right)$ with respect to the gauche population in the unstretched value $n_{g}\left(R_{0}\right)=65 \%$, at $\mathrm{T}=400 \mathrm{~K}$. This curve has been obtained by the histogram outlined in eq. (30) on the basis of the series 
of fixed- $f$ simulations for $N=512$ listed in table 1. The stretching variable used for the abscissa is chosen to test the Abe-Flory prediction $\left.\Delta n_{g}(R)=k\left(\left(R / R_{0}\right)^{2}-1\right)\right)$ with $\mathrm{k}=-0.656$ which is represented as a straight line in fig. [14]. We observe that Flory's theory predicts quite well the population shift up to $R \approx 3 R_{0}$ (corresponding to $X=R / L_{c} \approx 0.45$ for this chain length). The energetic part of the force clearly comes from the increased tendency for local bonds to go to lower torsional energy (trans state) as $\mathrm{R}$ increases.

\subsection{The superiority of the WLC model}

Data displayed in fig.1 and fig. 3 show respectively the variation of the reduced force $w(X)$ and the reduced free energy $W(X)$ as a function of $X=R / L_{c}$ for the two PE chain lengths investigated. In fig.11, we show the predicted curves $w_{F J C}(X)$ and $w_{W L C}(X)$ corresponding respectively to the two basic chain models while in fig. 3, only $W_{W L C}(X)$ is indicated. It turns out that, for the present PE case, the intermediate range of stretching is much better represented by the WLC model as it matches 'experimental' data up to a relative stretching of $\approx 85 \%$ for the force-extension law. At larger stretching, we note that the limiting behavior of the WLC model elasticity law $\left(L_{c}-R\right) \propto f^{-1 / 2}$ gives a too slow asymptotic evolution toward $R=L_{c}$. The same qualitative picture of elasticity has been observed in experiments on double-stranded DNA [6, 28]. We have however already noticed as the internal energy variation with stretching limits the validity of EOS of the type predicted by ideal models to the regime $X \in[0,0.65]$, narrower than is inferred by the force-extension behavior alone. This consideration puts some doubts on the present interpretation of the experimental elasticity laws through ideal models like FJC and WLC. We suggest that the validity of such models must be supported by internal energy versus extension curves.

In the interpretation of experimental data for DNA, the regime of extreme stretching $(X \geq 0.9)$ has been explained invoking a stretching dependent contour length $L_{c}(f)=$ $L_{c}^{0}\left(1+f / f_{0}\right)$, where $L_{c}^{0}$ is the contour length of the unstretched chain and $f_{0}$ is the elastic 
modulus of the chain [4, 29, 8, 7], to be used in connection with the elasticity law of the WLC model. The addition of the new parameter $f_{0}$, and its possible temperature dependence, allows the extended WLC model to overcome its previous limitation of predicting a constant $f_{e} / f$ ratio 30$]$. Although the qualitative feature of the departure of our MC data from the primitive WLC model prediction is very similar to that observed for double-stranded DNA, the elastic WLC model is of little relevance here since the contour length of our PE chain model, with rigid bonds and fixed bending angles, is constant.

We could instead define a stretching dependent persistence length $\ell_{p}(T, X) \equiv \ell_{p}^{0}(T)(1+$ $\Gamma(X, T))$, where $\ell_{p}^{0}(T)$ is the persistence length of the unstretched chain and $\Gamma(X, T)$ a generic function, to be used to represent the MC data in terms of the ideal WLC model

$$
\frac{2 f \ell_{p}^{0}(T)}{k_{B} T}=w(X, T)=\frac{w_{W L C}(X)}{(1+\Gamma(X, T))}
$$

Note that the temperature dependence of $\Gamma$ is necessary in order to obtain the observed deviation from a constant value for the ratios $\Delta E(X) / \Delta A(X)$ and $f_{E} / f$. Attempting this analysis with our data, we find that $\Gamma(X, T)$ remains very close to zero up to $X \simeq 0.6$, is slightly negative between $X=0.6$ and $X=0.85$ and then diverges to $\infty$ for larger $X$. Although in the intermediate regime, the temperature derivative of $\Gamma(X, T)$ could be invoked to explain the thermoelasticity behavior of our PE chain, the divergence of $\Gamma(X, T)$ at large $\mathrm{X}$ demonstrates the inadequacy of the WLC at very high stretching.

\section{A few remarks on the solvent effects on the thermo- elasticity properties}

In order to investigate the solvent quality effect on PE, we performed a series of experiments for $\mathrm{N}=256$ at $400 \mathrm{~K}$, but using $\lambda=0.01<<\lambda^{\star}(400)$ with the aim to model good solvent conditions at the same temperature. Excluded volume forces here modify the elasticity law as we showed earlier 18. 
In figure 5, we compare the $R$ dependence of the non local part of the internal energy for good and $\theta$ solvents. We already noticed in figure 2 that the sum of the repulsive and attractive energy contributions arising from non-local interactions is $R$ independent at $\theta$ point, a behavior we now show to be the result of subtle cancellation effects. Figure 5 shows the deviations $\Delta E^{r e p}(R, T)$ and $\Delta E^{\text {att }}(R, T)$ as defined in eq.(26) for the different solvent conditions. While at $\theta$ point, both terms exhibit linear dependence on $R$, the dependence is non linear in good solvent. These behaviors imply that the ratio $\left[-\Delta E^{r e p}(R, T) / \Delta E^{a t t}(R, T)\right]$, shown in the inset of figure 5, is constant in the whole range of stretching in $\theta$ solvent only. Therefore, this constant value 0.215 can be taken as an estimate of $\partial\left(\beta \lambda^{\star}\right) / \partial \beta$ which guarantees that the non local part of the internal energy does not depend on stretching. We note that this value used to produce data in figure 2 is in agreement with the prediction of Sariban et al. 23.

In good solvent, the contributions of repulsive and attractive non-local forces do not compensate anymore, whatever value is adopted for $\partial(\beta \lambda) / \partial \beta$. Assuming that $\lambda$ is nearly temperature independent in good solvent conditions, $\partial(\beta \lambda) / \partial \beta \approx \lambda$ which provides a monotonous decrease of internal energy with stretching (not shown). This indicates that dominant repulsive non local interactions disappear progressively as the size of tensile blobs characterized by self-avoiding walk statistics reduces with increasing force [1].

\section{Conclusions}

On the basis of a realistic chain model of polyethylene with solvent mediated non local interactions, the thermo-elastic properties of an individual chain at $\theta$ point and in good solvent conditions have been investigated by Monte-Carlo simulations sampling both fixed- $f$ and fixed- $R$ ensembles over a large stretching regime.

The $\theta$ state was previously obtained by adjusting an effective parameter in the attractive

part of the non-local interactions so that the chain size grows as $N^{1 / 2}$ at large $N$ 22, 23, 25. 
We have shown in section 6 that for such $\theta$ point conditions, the non-local interactions do not contribute to the average internal force over the whole stretching range. This property could be taken as an alternative criterion (using a single chain length) to search for $\theta$ point conditions for a particular polymer+solvent model. In good solvent conditions, non local interactions do contribute to the internal force for all stretching values.

We found that the thermo-elastic properties of the chain at $\theta$ point around $400 \mathrm{~K}$ in terms of $X=R / L_{c}$ are quite well represented, up to $X=65 \%$, by an EOS where the temperature and relative elongation dependencies are factorized, namely $f(X, T)=k_{B} T w(X) / \ell_{p}(T)$, where $\ell_{p}$ is the persistence length of the chain determined from free chain simulations 25]. From the variation of the internal energy and free energy differences with stretching, we inferred the value of the temperature derivative of $\ell_{p}$, namely $d \ln \left(\ell_{p}\right) / d \ln (T)=-0.42$ in fair agreement with experimental observations and theoretical predictions for PE chains at $\theta$ point. We point out here that this temperature variation implies that the internal force at any stretching has a non negligible enthalpic part which is negative because extension drives more and more dihedral angles to adopt the lowest energy trans state.

Up to $65 \%$, the $X$ dependencies of the force, i.e. the function $w(X)$ above (see eq. (河)), and the of internal energy difference (see eq. (32)) turn out to be very well represented by the WLC model, using the contour length and persistence length of our atomistic PE model at equilibrium, without recourse to any adjustable parameter. By comparison, the FJC model appears to be much less representative of the PE finite extensibility effects. This result may be useful to interpret AFM stretching experiments for which the minimal force which can be detected is above the crossover force between the scaling regime and the finite extensibility regime. We stress again here that the validity of the WLC model, in the present context, does not imply that the internal force has a purely entropic character.

Ad-hoc extensions of ideal chain models which have been proposed so far in the literature do not seem to apply to the highest stretching regime explored here $(65 \%<X<90 \%)$. The 
general question of thermodynamic consistency of these "elastic" WLC and FJC models should be carefully analyzed before they could be considered something more than simple fitting functions. In particular, if stretching is measured at a single temperature, the elasticity law $f(X, T)$ and the internal energy $E(X, T)$ must be simultaneously considered because thermodynamic consistency (see eq.(9))directly relates the change of the internal force with temperature (at constant $X$ ) to the change of internal energy with stretching (at constant $T)$.

\section{Acknowledgment}

J.T.T. gratefully acknowledges financial support from the BOF-NOI UA 2001 and the Flemish Region-I.W.T. 
[1] P. G. de Gennes, Scaling Concepts in Polymer Physics (Cornell Univ. Press, Ithaca, 1979).

[2] P. Pincus, Macromolecules 9, 386 (1976).

[3] I. Webman, J. L. Lebowitz, and M. H. Kalos, Phys. Rev. A 23, 316 (1981).

[4] T. Odijk Macromolecules 28, 7016 (1995).

[5] S. B. Smith, L. Finzi, and C. Bustamante, Science 258, 1122 (1992).

[6] J.F. Marko and E.D. Siggia, Macromolecules 28, 8759 (1995).

[7] R. Merkel, Physics Report 346, 343 (2001).

[8] A. Janshoff, and M. Neitzert, and Y. Oberdörfer, and H. Fuchs, Angew. Chem. Int. Ed. 39,3212 (2000).

[9] P. J. Flory, Statistical Mechanics of Chain Molecules (Hanser Publ., Munich,1989).

[10] A.T. Boothroyd, A.R. Rennie, and C.-B. Boothroyd, Europhys. Lett. 15, 715 (1991).

[11] C. Ortiz and G. Hadziioannou, Macromolecules 32, 780 (1999).

[12] C. Bouchiat,M.D. Wang, J.-F. Allemand, T. Strick, S.M. Block, and V. Croquette, Biophysical Journal 76, 409 (1999).

[13] S. Yamamoto,Y. Tsujii, and T. Fukuda, Macromolecules 33, 5995 (2000).

[14] Y. Abe, and P.J. Flory, J. Chem. Phys. 52, 2814 (1970).

[15] P. Cifra and T. Bleha, J. Chem. Soc. Faraday Trans. 91, 2465 (1995).

[16] M.W. Konrad and J.I. Bolonick J. Am. Chem Soc. 118, 10989 (1996). 
[17] C. Pierleoni, G. Arialdi, and J.-P. Ryckaert, Phys. Rev. Lett. 79, 2990 (1997).

[18] J. T. Titantah, C. Pierleoni, and J.-P. Ryckaert, Phys. Rev. E 60, 7010 (1999).

[19] R.G. Maurice, and C.C. Matthai, Phys. Rev. E 60, 3165 (1999).

[20] K.M. Kosikov, A.A. Gorin, V.B. Zhurkin and W.K. Olson J. Mol. Biol. 289, 1301 (1999).

[21] B. Heymann, and H. Grubmüller, Chem. Phys. Lett. 307, 425 (1999).

[22] A. Sariban, J. Brickmann, J. van Ruiten and R.J. Meier, Macromolecules 25, 5950 (1992).

[23] A. Sariban, T. Mosell, and J. Brickmann, Macrom. Theory and Simul. 3, 963 (1994).

[24] J. Smith, and M. Karplus, J. Am. Chem. Soc. 114, 801 (1992).

[25] M. Destrée, A. Lyulin, and J.P. Ryckaert, Macromolecules 29, 1721 (1996)

[26] D. Frenkel and B. Smit, Understanding Molecular Simulation (Academic Press, San Diego, 1996).

[27] R.M. Neumann, Phys. Rev. A 34, 3486 (1986)

[28] R. H. Austin, J. P. Brody, E. C. Cox, T. Duke, and W. Volkmuth, Phys. Today, 50, 32 (1997).

[29] M. Rief, J.M. Fernandez, and H.E. Gaub Phys. Rev. Lett. 81, 4764 (1998).

[30] A force dependent contour length in eq.(4) or eq.(5), although physically soundable, transform the equations in implicit ones for the force. From these equation it is difficult to infer the thermodynamics. We could however think to a generic temperature dependence of the contour length through the temperature dependence of the extra 
parameter. For this elastic WLC model with temperature dependent persistence length $\ell_{p}(T)$ and contour length $L_{c}(T)$ we have the following EOS

$$
\frac{2 f \ell_{p}(T)}{k_{B} T}=w_{W L C}(X(T))
$$

which implies the following relation for the thermoelasticity

$$
\frac{\Delta E}{k_{B} T}=\left[\frac{d \ln \ell_{p}}{d \ln T}-\frac{d \ln L_{c}}{d \ln T}\right] \frac{\Delta A}{k_{B} T}+\frac{L_{c} d \ln L_{c}}{\ell_{p} d \ln T} X w_{W L C}(X(T))
$$

more general than the simple eq.(32). 


\section{Figures and tables captions}

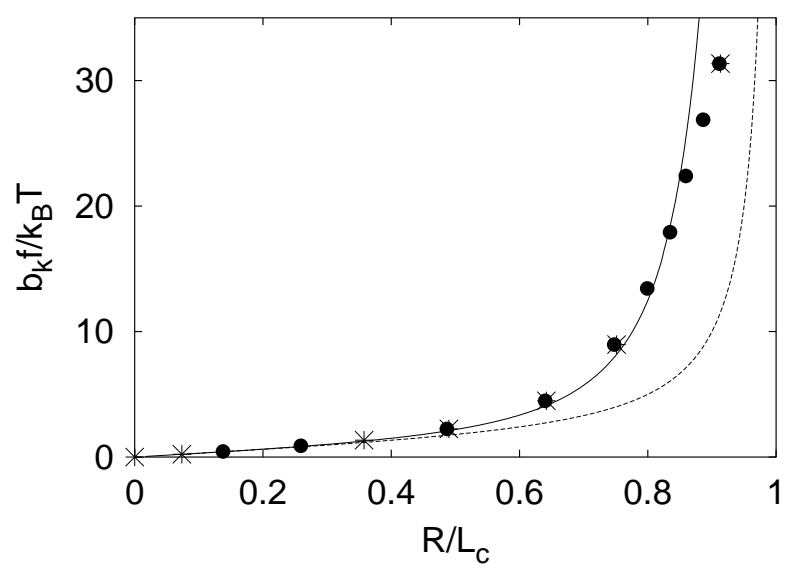

Fig. 1: Force-extension relationships obtained in the fixed- $f$ ensemble for $\mathrm{N}=256$ (closed circles) and $\mathrm{N}=512$ (stars). Error bars are smaller than the size of the symbols. Continuous and dashed lines represent the elasticity law of the WLC and the FJC models respectively. 


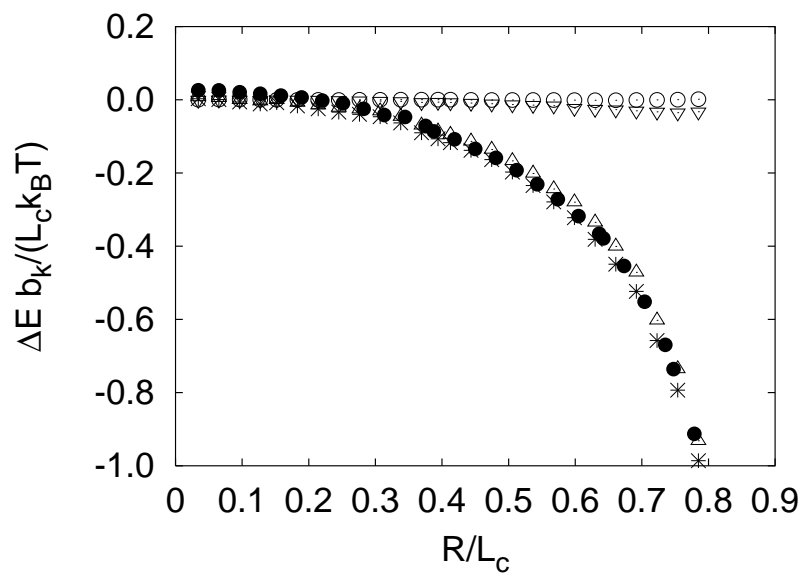

Fig. 2: Reduced internal energy difference $\Delta E b_{k} / L_{c} k_{B} T$ versus the relative extension $X=$ $R / L_{c}$ for a $\mathrm{PE}$ chain at $\theta$ point $(400 \mathrm{~K})$ of $\mathrm{N}=256$ (closed circles) and $\mathrm{N}=512$ (stars). For $\mathrm{N}=512$, we also show the various contributions to the reduced internal energy namely the torsional energy (upward triangles) the 1-5 local interactions (downward triangle) and the non local interaction (open circles). 


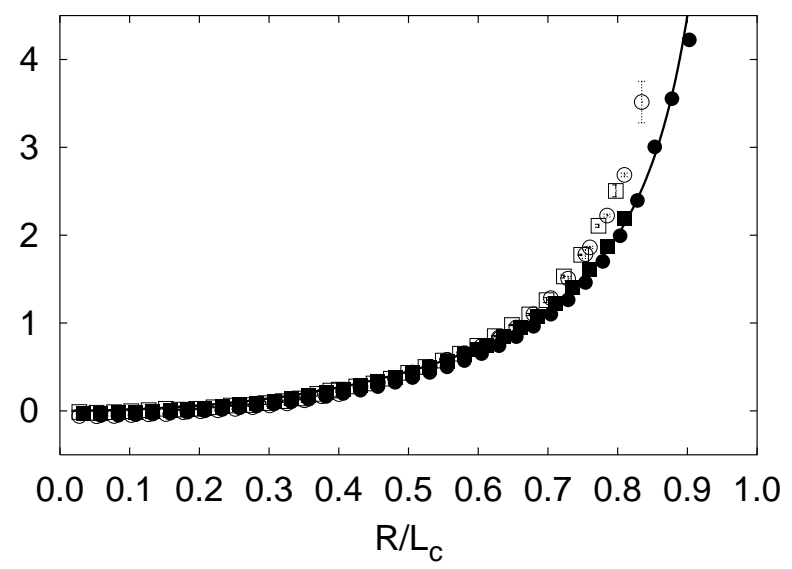

Fig. 3: Comparison between $\Delta A(R)\left(\ell_{p} / L_{c} k_{B} T\right) \quad$ (closed symbols) and $\Delta E(R)\left(\ell_{p} / L_{c} k_{B} T\right)\left(d \ln \left(\ell_{p}\right) / d \ln (T)\right)^{-1}$ (open symbols) for $\mathrm{N}=256$ (circles) and $\mathrm{N}=512$ (squares) versus the relative stretching $R / L_{c}$. The value $\left[d \ln \left(\ell_{p}\right) / d \ln (T)\right]=-0.42$ has been adopted to rescale the internal energy difference. The continuous curve represents $W_{W L C}(x)$ as defined in the text. 


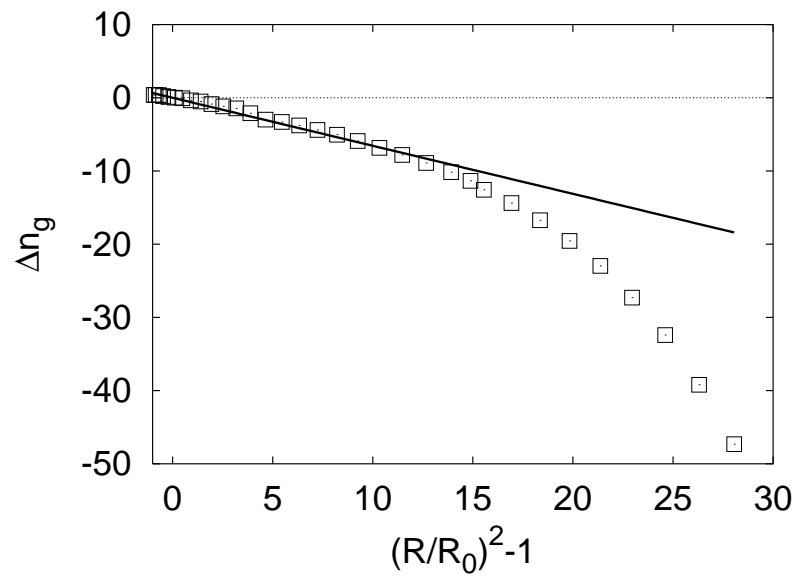

Fig. 4: Percentage of gauche population with respect to the unstretched state $\Delta n_{g}=n_{g}(x)-$ $n_{g}(0)$ in the fixed- $R$ ensemble as a function of $\left(R / R_{0}\right)^{2}-1$ for a $\mathrm{N}=512$ chain at $\theta$ point. The straight line is the Flory's prediction for small stretching. 


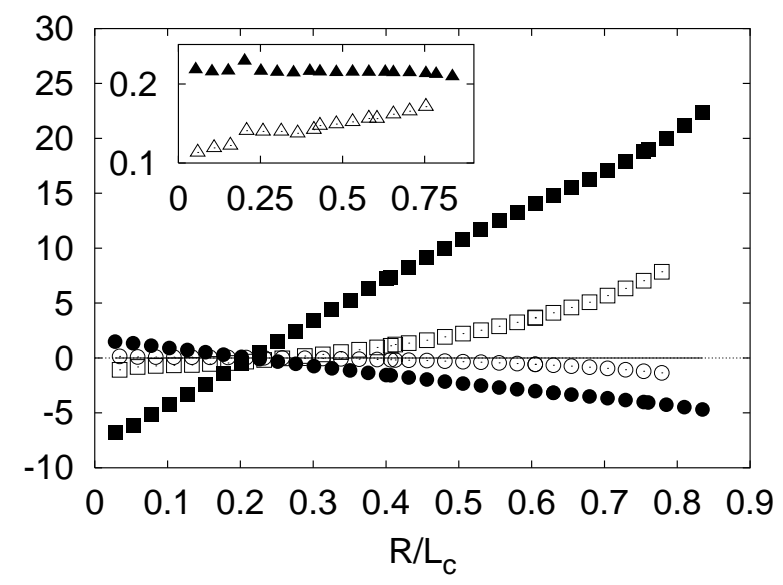

Fig. 5: Attractive (squares) and repulsive (circles) contributions of the non local part of the energy with respect to the unstretched state in good solvent (open symbols) and $\theta$ solvent (closed symbols) at $400 \mathrm{~K}$. Energies are normalized to $k_{B} T$. In the inset we show the ratio of minus the repulsive part to the attractive part which is constant for $\theta$ solvent but depends on stretching for good solvent. 
Table 1: List of MC experiments in the fixed- $f$ ensemble for two different system sizes $N$ at $\theta$ point. The last column gives the range of extensions statistically accessible, for the given imposed force $f$ and number of statistically independent configurations of the chain.

\begin{tabular}{|c|c|c|c|}
\hline$N$ & imposed $f$ & \# indep. configurations & range of R/Lc exploited \\
\hline \hline 256 & 0.0 & 73100 & $0.00-0.25$ \\
\hline & 0.2 & 180400 & $0.25-0.47$ \\
\hline & 0.5 & 153500 & $0.47-0.62$ \\
\hline & 1.0 & 95400 & $0.62-0.75$ \\
\hline & 2.0 & 27000 & $0.75-0.81$ \\
\hline & 3.0 & 27400 & $0.81-0.87$ \\
\hline & 4.0 & 25000 & $0.87-0.92$ \\
\hline 512 & 0.0 & 36000 & $0.00-0.28$ \\
\hline & 0.3 & 35000 & $0.28-0.51$ \\
\hline & 0.5 & 23000 & $0.51-0.60$ \\
\hline & 1.0 & 24000 & $0.60-0.71$ \\
\hline & 2.0 & 19200 & $0.71-0.81$ \\
\hline
\end{tabular}




\section{Figure captions}

Figure 1: Force-extension relationships obtained in the fixed- $f$ ensemble for $\mathrm{N}=256$ (closed circles) and $\mathrm{N}=512$ (stars). Error bars are smaller than the size of the symbols. Continuous and dashed lines represent the elasticity law of the WLC and the FJC models respectively.

Figure 2: Reduced internal energy difference $\Delta E b_{k} / L_{c} k_{B} T$ versus the relative extension $X=R / L_{c}$ for a $\mathrm{PE}$ chain at $\theta$ point $(400 \mathrm{~K})$ of $\mathrm{N}=256$ (closed circles) and $\mathrm{N}=512$ (stars). For $\mathrm{N}=512$, we also show the various contributions to the reduced internal energy namely the torsional energy (upward triangles) the 1-5 local interactions (downward triangle) and the non local interaction (open circles).

Figure 3: Comparison between $\Delta A(R)\left(\ell_{p} / L_{c} k_{B} T\right)$ (closed symbols) and $\Delta E(R)\left(\ell_{p} / L_{c} k_{B} T\right)\left(d \ln \left(\ell_{p}\right) / d \ln (T)\right)^{-1}$ (open symbols) for $\mathrm{N}=256$ (circles) and $\mathrm{N}=512$ (squares) versus the relative stretching $R / L_{c}$. The value $\left[d \ln \left(\ell_{p}\right) / d \ln (T)\right]=-0.42$ has been adopted to rescale the internal energy difference. The continuous curve represents $W_{W L C}(x)$ as defined in the text.

Figure 4: Percentage of gauche population with respect to the unstretched state $\Delta n_{g}=n_{g}(x)-n_{g}(0)$ in the fixed- $R$ ensemble as a function of $\left(R / R_{0}\right)^{2}-1$ for a $\mathrm{N}=512$ chain at $\theta$ point. The straight line is the Flory's prediction for small stretching.

Figure 5: Attractive (squares) and repulsive (circles) contributions of the non local part of the energy with respect to the unstretched state in good solvent (open symbols) and $\theta$ solvent (closed symbols) at 400K. Energies are normalized to $k_{B} T$. In the inset we show the ratio of minus the repulsive part to the attractive part which is constant for $\theta$ solvent but depends on stretching for good solvent. 